\title{
A simple merging algorithm for jet angular correlation studies
}

\section{Junya Nakamura}

Institute for Theoretical Physics, University of Tübingen, Auf der Morgenstelle 14, 72076 Tübingen, Germany

E-mail: junya.nakamura@uni-tuebingen.de

ABSTRACT: A tree level merging algorithm which guarantees the leading order (LO) accuracy of angular correlations among jets is proposed and studied. The algorithm is designed so that $n$-jet events are generated exclusively according to the LO $n$-parton production cross section and each of the $n$ jets is close to each of the $n$ partons in terms of the jet measure. As a result, the kinematics of each of the $n$ jets strictly follows that of each of the $n$ partons and the LO accuracy of angular correlations among the $n$ jets is preserved. The validity of the method as a merging algorithm is confirmed by comparing it against existing merging algorithms for several observables in the top quark pair production. Correlations in the azimuthal angle difference between the two highest transverse momentum jets with large rapidity separations are evaluated as examples.

Keywords: Monte Carlo Simulations, Jets

ARXIV EPRINT: 1509.04164 


\section{Contents}

1 Introduction 1

2 Algorithm 3

3 Numerical studies $\quad 8$

3.1 Setup and the cross section 8

$\begin{array}{lll}3.2 & \text { Distributions } & 10\end{array}$

4 Summary and discussion $\quad 16$

\section{Introduction}

Angular correlations among jets produced together with heavy particles have been studied actively for a long time, because they can provide important information about the heavy particles [1-7]. For instance it has been shown that the distribution of the azimuthal angle difference $\Delta \phi=\phi_{1}-\phi_{2}$ between two partons in the gluon fusion production of a Higgs boson plus the two partons is very sensitive to a charge-conjugation and parity $(\mathrm{CP})$ property of the Higgs boson [1-5]. By observing the $\Delta \phi$ distribution and comparing it with theoretical predictions, we can measure $\mathrm{CP}$ violation in the Higgs sector [3, 4].

In order to read the information of heavy particles from angular correlations among jets produced in association with them, it will be necessary to produce the accurate predictions of observables, such as $\Delta \phi$, which measure the angular correlations. Tree level merging algorithms [8-22], which combine leading order (LO) cross sections for multiparton final state with the parton shower, are nowadays standard tools used for simulating processes including multijet final state. Models of the parton shower base the Dokshitzer-GribovLipatov-Altarelli-Parisi (DGLAP) evolution equation [23-25] and thus the parton shower guarantees the leading logarithmic (LL) accuracy for the kinematics of produced partons. Therefore, the accuracy guaranteed in merging algorithms can be seen as the LO plus LL. The virtue of merging algorithms is that they can combine LO cross sections smoothly with the LL parton shower so that the dependence on an artificial scale at which they are combined is minimised, and produce fully inclusive event samples.

A LO multi-parton production cross section predicts angular correlations among the produced partons at the LO accuracy, while the LL parton shower does not have ability to predict angular correlations among any partons. Considering this fact, when our objective is to predict angular correlations among constructed jets, the accuracy minimally required for the kinematics of the jets should be the LO. If the kinematics of a jet is determined or largely influenced by the LL parton shower during a merging procedure, it hardly has the LO accuracy and hence it is not appropriate to use the event containing this jet. Merging 
algorithms in the literature potentially have the ambiguity in the accuracy of jets, namely it is not necessarily clear whether the kinematics of a jet constructed by clustering particles in final state after a merging procedure has the LO accuracy or not. This is because their virtue is smooth combination of LO cross sections and the LL parton shower.

In ref. [26], the azimuthal angle difference $\Delta \phi=\phi_{1}-\phi_{2}$ between the two highest transverse momentum $p_{T}$ jets with a large rapidity separation in the $t \bar{t}$ production is studied by using the CKKW-L merging algorithm [9, 12, 20] with the parton shower model [27-29] in PYTHIA8 [30, 31]. There, it is found that the correlation between the two jets can be lost in a non-negligible fraction of the events when the LO cross sections for the $t \bar{t}+0,1,2$ parton are merged with the parton shower, because a jet originating from the parton shower has a higher $p_{T}$ than one of the two jets originating from the two partons of the LO cross section. This shows one example of the case that the kinematics of a jet is determined or largely influenced by the LL parton shower during a merging procedure. Although it is also found that the loss of the correlation can be avoided by merging the LO $t \bar{t}+3$ parton cross section additionally, calculating LO cross sections for higher multiplicity is time-consuming, thus we want to avoid it.

In this work, I construct a merging algorithm which does guarantee the LO accuracy of angular correlations among jets and hence does not have the above ambiguity. The algorithm proceeds in a similar way as the MLM $[13,14]$ and the $k_{\perp}$-jet MLM $[14,16]$ algorithms. The difference from these existing algorithms is that the following idea is implemented:

- The definition of jets used during a merging procedure is set identical to the one used during physics analyses of jets.

As a result, the $n$-jet events are generated exclusively according to the LO $n$-parton production cross section and furthermore each of the $n$ jets is close (matched) to each of the $n$ partons in terms of the jet measure. The kinematics of each of the $n$ jets strictly follows that of each of the $n$ partons and thus the LO accuracy of angular correlations among the $n$ jets is preserved. When $n=n_{\max }$, where $n_{\max }$ denotes the maximal number of partons produced by a LO cross section, the LO $n_{\max }$-parton production cross section generates the inclusive $n_{\max }$-jet events $\left(n_{\text {jets }} \geq n_{\max }\right.$, where $n_{\text {jets }}$ denotes the number of jets) and each of the $n_{\max }$ highest $p_{T}$ jets is matched to each of the $n_{\max }$ partons in terms of the jet measure. Therefore, this algorithm produces fully inclusive event samples as other existing merging algorithms. In the produced inclusive event samples, the kinematics of each of up to the $n_{\max }$ highest $p_{T}$ jets strictly follows that of each of the LO matrix-element partons. Therefore, as long as the highest $p_{T}$ jets are always picked up, the LO accuracy of angular correlations among up to $n_{\max }$ jets is preserved.

The MLM and the $k_{\perp}$-jet MLM algorithms use the traditional cone algorithm and the exclusive $k_{\perp}$-jet algorithm [32], respectively, as a clustering algorithm, and parameters that the clustering algorithm contains are chosen independently of the definition of jets used during analyses of jets. In other words, the definition of jets used at the analysis level is nothing to do with the merging setup in these merging algorithms. In the new merging algorithm, the merging setup is determined according to the definition of jets used 
at the analysis level. This might sound in conflict with the strategy of merging algorithms. It is described in ref. [13] that a good merging algorithm should produce fully inclusive event samples and any definition of jets can be used at the analysis level of the produced event samples. However, when the LO accuracy is strictly required for the kinematics of each jet, the merging setup can be determined only after the definition of jets used at the analysis level is fixed, as in the new algorithm. If the definition of jets is changed, the new inclusive event samples have to be generated with the merging setup corresponding to the new definition of jets.

The $t \bar{t}$ production in proton-proton collisions is simulated by using the new merging algorithm with $n_{\max }=2,3$. Several observables and quantities, including the azimuthal angle differences $\Delta \phi=\phi_{1}-\phi_{2}$ between the two highest $p_{T}$ jets with vector boson fusion cuts as an angular correlation observable, are studied. The results are compared with those of other existing algorithms, the CKKW-L and the MLM algorithms. It is found that the new algorithm produces quite similar results with the MLM algorithm, when jets are defined by the anti- $k_{T}$ algorithm [33]. As to $\Delta \phi$, the CKKW- $\mathrm{L}_{n_{\max }=2}$ results consistently show visible discrepancies from the other results near the bottom region $(|\Delta \phi| \sim 0)$ and the peak region $(|\Delta \phi| \sim 2)$, while all the other results agree well with each other.

In section 2, the new merging algorithm and an event generation procedure according to it are described in detail. In section 3 , the results of the simulation are presented. In section 4 , I summarise my findings.

\section{Algorithm}

In this section, the ideas of the new merging algorithm and the merging procedure are described in detail.

The new algorithm is constructed with a goal of eliminating the ambiguity in the accuracy of jets which potentially exists in merging algorithms as discussed in section 1 and guaranteeing the leading order (LO) accuracy of angular correlations among jets. The algorithm proceeds based on the same philosophy of the MLM [13, 14] algorithm that a parton shower evolution of a matrix-element parton should not change the kinematics of the parton significantly and the event which does not satisfy this condition is vetoed. This vetoing procedure effectively corresponds to the calculation of the Sudakov form factors in the CKKW $[8,10,17,18]$ and the CKKW-L algorithms $[9,12,20]$. The following idea is implemented in the new algorithm,

- The definition of jets used during a merging procedure is set identical to the one used during physics analyses of jets.

As a result, the generated event samples achieve the following:

The $n$-jet events are generated exclusively according to the LO n-parton production cross section and furthermore each of the $n$ jets is close to each of the $n$ partons in terms of the jet measure. 
The virtue of this achievement is that the kinematics of each of the $n$ jets strictly follows that of each of the matrix-element $n$ partons. The LO accuracy of angular correlations among the $n$ jets is also guaranteed. In order for the generated event samples to be fully inclusive, we make them further satisfy the following:

The inclusive $n_{\max }$-jet events are generated exclusively according to the LO $n_{\max }$-parton production cross section and furthermore each of the $n_{\max }$ highest transverse momentum jets is close to each of the $n_{\max }$ partons in terms of the jet measure.

Here $n_{\max }$ denotes the maximal number of partons produced by a LO cross section. The kinematics of each of the $n_{\max }$ highest $p_{T}$ jets strictly follows that of each of the matrixelement $n_{\max }$ partons. Therefore, in the generated fully inclusive event samples, as long as the highest transverse momentum $p_{T}$ jets are always picked up, the LO accuracy of angular correlations among up to $n_{\max }$ jets is preserved. The kinematics of the additional jets (jets softer than the $n_{\max }$ highest $p_{T}$ jets in terms of $p_{T}$ ) is determined by the leading logarithmic (LL) parton shower, hence they do not have correct angular correlations with any other jets.

In my numerical studies given in the following section, the anti- $k_{T}$ algorithm [33] is chosen as a jet clustering algorithm when distributions of jets are studied. Therefore, it is also used during the merging procedure in the new merging algorithm. Below I describe the merging procedure in detail by focusing on the case of using the anti- $k_{T}$ algorithm. Differences induced by the use of other clustering algorithms are also discussed.

Let us consider merging the event samples $X+0,1,2, \cdots, n_{\max }$-parton produced by the LO cross sections, where $X$ denotes a heavy object such as a $\mathrm{Z}$ boson or $t \bar{t}$, with the parton shower. The soft and collinear divergences in the LO cross sections are regularised by a generation cutoff $Q_{\mathrm{cut}}^{\mathrm{ME}}$. The definition of $Q_{\text {cut }}^{\mathrm{ME}}$ has to respect the definition of jets used in the merging procedure (thus used at the analysis level, too, in the new algorithm). Jets are defined by a clustering algorithm and parameters that the algorithm contains. The anti- $k_{T}$ algorithm basically contains two parameters which we can choose their values freely, the radius parameter $R^{\text {jet }}$ and the lower $p_{T}$ cutoff on jets $p_{T \text { cut }}^{\text {jet }}$. Hence $Q_{\text {cut }}^{\mathrm{ME}}$ should be defined by

$$
\begin{array}{r}
\Delta R_{i j}=\sqrt{\left(y_{i}-y_{j}\right)^{2}+\left(\phi_{i}-\phi_{j}\right)^{2}}>R_{\mathrm{cut}}^{\mathrm{ME}}, \\
p_{T i}>p_{T \mathrm{cut}}^{\mathrm{ME}},
\end{array}
$$

where $p_{T i}, y_{i}$ and $\phi_{i}$ are the $p_{T}$, rapidity and azimuthal angle of outgoing parton $i$. Imposing cutoffs on the rapidity of partons is not needed. This generation cutoff in eq. (2.1) is the same as the one used in the MLM algorithm which uses the traditional cone jet clustering algorithm. The inclusive $k_{T}$ algorithm [34] and the inclusive $p p$ Cambridge/Aachen algorithm $[35,36]$ contain the same two parameters with the anti- $k_{T}$ algorithm. Therefore, the generation cutoff $Q_{\mathrm{cut}}^{\mathrm{ME}}$ should also be defined by eq. (2.1), when one of these two clustering algorithms is used. The exclusive $k_{T}$ algorithm [32] introduces the radius parameter $R^{\text {jet }}$ 
and a scale $d_{\text {cut }}^{\text {jet }}$. The generation cutoff $Q_{\text {cut }}^{\mathrm{ME}}$ should be defined by

$$
\begin{aligned}
d_{i j}=\min \left(p_{T i}, p_{T j}\right) \Delta R_{i j} / R_{\mathrm{cut}}^{\mathrm{ME}} & >d_{\mathrm{cut}}^{\mathrm{ME}}, \\
p_{T i} & >d_{\text {cut }}^{\mathrm{ME}} .
\end{aligned}
$$

This generation cutoff is used in the $k_{\perp}$-jet MLM algorithm, since the $k_{\perp}$-jet MLM algorithm uses the exclusive $k_{T}$ algorithm during the merging procedure. In order to avoid missed phase space, the generation cutoff must satisfy

$$
p_{T \text { cut }}^{\text {jet }} \geq p_{T \text { cut }}^{\mathrm{ME}}, \quad R^{\text {jet }} \geq R_{\text {cut }}^{\mathrm{ME}}, \quad d_{\text {cut }}^{\text {jet }} \geq d_{\text {cut }}^{\mathrm{ME}} .
$$

An event sample of the $X+n$-parton, where $n<n_{\max }$, is treated as follows. A parton shower program is executed on the event sample. Once the shower evolution is performed until the shower cutoff scale, all final state partons within a rapidity range $|y|<y_{\text {cut }}^{\text {clus }}$ are clustered to construct jets according to the anti- $k_{T}$ algorithm with $R^{\text {jet }}$ and $p_{T \text { cut }}^{\text {jet }}$. If the number of the constructed jets $n_{\text {jets }}$ is not identical to $n$, the event sample is vetoed. If the event sample survives $\left(n_{\text {jets }}=\mathrm{n}\right)$, the distance parameters $\Delta R$ defined in eq. (2.1a) between the $n$ jets and the $n$ partons are calculated, and then it is checked whether the following relation is satisfied between each of the $n$ jets and each of the $n$ partons, or not

$$
\Delta R_{\text {jet, parton }}<C_{\text {match }} \times R^{\text {jet }},
$$

where $C_{\text {match }}$ is a factor of $O(1)$ and its explanation is given in the next paragraph. If the jet satisfies the above relation with the parton, it means that the jet is close to the parton in terms of the measure of the anti- $k_{T}$ algorithm. The jet is called matched with the parton. If the matching between the jet and the parton is confirmed for all of the $n$ jets and all of the $n$ partons, then the event sample is accepted. Once the event sample is accepted, all final state partons are again clustered to construct jets which are defined in the same way as above, but this time only those within a rapidity range $|y|<y_{\text {cut }}^{\text {detect }}$ are clustered. A value for $y_{\text {cut }}^{\text {dete }}$ should reflect an actual experimental detector and can be different from $y_{\text {cut }}^{\text {clus }}$. The following relation should be satisfied between the two cutoffs,

$$
y_{\text {cut }}^{\text {detect }} \leq y_{\text {cut }}^{\text {clus }} .
$$

The constructed jets will be used for physics analyses. If the values for $y_{\text {cut }}^{\text {clus }}$ and $y_{\text {cut }}^{\text {detect }}$ are different, the jets constructed at this stage can also be different from those constructed before and therefore $n_{\text {jets }} \neq n$ and/or the jets are not necessarily matched with the $n$ partons in some of the events. However, when $y_{\text {cut }}^{\text {detect }}$ is large enough so that there is only a small fraction of the events of the $X+n$-parton above $y_{\text {cut }}^{\text {dete }}$, the difference is expected to be small. When the inclusive $k_{T}$ algorithm or the inclusive $p p$ Cambridge/Aachen algorithm is used for the clustering after the parton shower evolution, the check of the matching between the jet and the parton can be performed by using the same equation in eq. (2.4). If the exclusive $k_{T}$ algorithm with $R^{\text {jet }}$ and $d_{\text {cut }}^{\text {jet }}$ is used, the matching should be checked by using the following relation:

$$
\min \left(p_{T \text { jet }}, p_{T \text { parton }}\right) \Delta R_{\text {jet, parton }} / R^{\text {jet }}<C_{\text {match }} \times d_{\text {cut }}^{\text {jet }} .
$$


The same relation with $C_{\text {match }}=1$ is used in the $k_{\perp}$-jet MLM algorithm. An event sample of the $X+n_{\max }$-parton is treated in a different way. After the parton shower evolution of the event sample and the subsequent jet clustering in the same way as in the event samples of the $X+n$-parton $\left(n<n_{\max }\right)$, the event sample is vetoed if the number of the constructed jets $n_{\text {jets }}$ is less than $n_{\max }\left(n_{\text {jets }}<n_{\max }\right)$. If the event sample survives $\left(n_{\text {jets }} \geq n_{\text {max }}\right)$, the matching between the jets and the partons is checked by using the same matching measure in eq. (2.4). If the $n_{\max }$ highest $p_{T}$ jets are matched with the $n_{\max }$ partons, the event sample is accepted.

The parameter $C_{\text {match }}$ in eqs. (2.4) and (2.6) is an important parameter, since it determines how far away the jet is allowed to be from the matrix-element parton. In addition to this, the Sudakov suppression can depend on it. The implementations of the MLM algorithm in Alpgen [13, 14] and in MadGraph5_aMC@NLO [37] use a constant value $C_{\text {match }}=1.5$. However, there is nothing that uniquely determines $C_{\text {match }}$. This is considered as a tuning parameter and even non-constant values may be possible. In my implementation of the new merging algorithm, the same constant value $C_{\text {match }}=1.5$ is chosen.

Below I describe event generation steps for completeness. The numerical study in section 3 is performed according to these.

1. Generate the event samples for the $X+0,1, \ldots, n_{\max }$-parton production processes at proton-proton (pp) collisions according to the LO cross sections, where $X=t \bar{t}$ and $n_{\max }=2$ or 3 . The soft and collinear singularity is regularised by imposing the generation cutoff in eq. (2.1). A fixed value $t_{\Lambda}=20 \mathrm{GeV}$ is used for the scales in the strong couplings $\alpha_{s}$ and in the parton distribution functions (PDFs).

2. Select an event sample for the $X+n$-parton process, where $n=0,1, \cdots, n_{\max }$, with the probability proportional to its integrated LO cross section obtained in the step 1 ,

$$
P_{n}=\frac{\sigma(p p \rightarrow X+n)}{\sum_{i=0}^{n_{\max }} \sigma(p p \rightarrow X+i)} .
$$

3. Construct a PYTHIA8 parton shower history of the event sample by successively clustering two partons into one parton. This is done by doing the exact inverse of the shower generation [27-29] of PYTHIA8. If I let $\{p\}_{X+n}$ denotes a complete specification of the event sample, the history consists of intermediate events $\{p\}_{X+(n-1)},\{p\}_{X+(n-2)}, \cdots,\{p\}_{X+i}, \cdots,\{p\}_{X+1},\{p\}_{X}$ with the ordered clustering scales $t_{n}<t_{n-1}<\cdots<t_{i+1}<\cdots<t_{2}<t_{1}$. Below I give some technical details in the history construction:

- The clustering $2 \rightarrow 1$ must respect the QCD $1 \rightarrow 2$ vertices and an intermediate event after the clustering must be one of the possible configurations. For instance, the lowest order configuration $\{p\}_{X}$ must be either $g g \rightarrow t \bar{t}$ or $q \bar{q} \rightarrow t \bar{t}$ where $q$ and $\bar{q}$ have the same flavour.

- Sequential clustering scales are required to be ordered, from lower scales to higher scales. 
- If there are more than one candidates for a clustering pair at a clustering step, the one which has the lowest clustering scale is always chosen.

- In some events, the shower history construction stops because (1) an intermediate event cannot have one of the possible configurations or (2) a clustering pair which gives a higher scale than the previous clustering scale cannot be found. In such an event, a shower history is not constructed and the PDF and $\alpha_{s}$ weight in the step 4 is calculated differently.

4. Calculate the PDF and $\alpha_{s}$ weight based on the parton shower history. Let us define the energy fractions and the parton types of the incoming partons in the $\{p\}_{X+i}$ by $x_{1}^{(i)}, x_{2}^{(i)}$ and $f_{1}^{(i)}, f_{2}^{(i)}$, respectively. The weight for the $\{p\}_{X+i}$ is given by [20]

$$
f\left(z_{i+1}, t_{i+1} ;\{p\}_{X+i}\right)=\frac{\alpha_{s}\left(t_{i+1}\right)}{\alpha_{s}\left(t_{\Lambda}\right)} \frac{f_{1}^{(i)}\left(x_{1}^{(i)}, t_{i}\right)}{f_{1}^{(i)}\left(x_{1}^{(i)}, t_{i+1}\right)} \frac{f_{2}^{(i)}\left(x_{2}^{(i)}, t_{i}\right)}{f_{2}^{(i)}\left(x_{2}^{(i)}, t_{i+1}\right)},
$$

from which the total weight for the event sample $\{p\}_{X+n}$ is

$$
\prod_{i=0}^{n} f\left(z_{i+1}, t_{i+1} ;\{p\}_{X+i}\right)
$$

where $t_{0}=t_{X}$ (defined below) and $t_{n+1}=t_{\Lambda}$ (defined in the step 1). The $\alpha_{s}$ value in eq. (2.8) is set identical to the one used in the shower evolution of PYTHIA 8 and is $\alpha_{s}\left(m_{z}\right)=0.1365$ in PYTHIA8 version 8212. The scale $t_{X}$ is determined from the constructed lowest order configuration $\{p\}_{X}$ and is defined by

$$
t_{X}^{2}=E_{T}^{2}(t)+E_{T}^{2}(\bar{t}),
$$

where $E_{T}(t)$ is the transverse energy $E_{T}^{2}=m^{2}+p_{T}^{2}$ of the top quark and $E_{T}(\bar{t})$ is that of the anti-top quark. This scale $t_{X}$ is also used as the scale of the strong couplings $\alpha_{s}^{2}$ for the lowest order configuration,

$$
\frac{\alpha_{s}^{2}\left(t_{X}\right)}{\alpha_{s}^{2}\left(t_{\Lambda}\right)}
$$

This is added as a multiplicative factor in eq. (2.9). $\alpha_{s}\left(m_{z}\right)=0.13$ is used in this factor. If the parton shower history of the event sample is not constructed in the step 3, the PDF and $\alpha_{s}$ weight is calculated based only on the information of the generated event $\{p\}_{X+n}$ :

$$
\frac{\alpha_{s}^{2}\left(t_{X}\right)}{\alpha_{s}^{2}\left(t_{\Lambda}\right)} \frac{\alpha_{s}\left(E_{T}(1)\right) \alpha_{s}\left(E_{T}(2)\right) \cdots \alpha_{s}\left(E_{T}(n)\right)}{\alpha_{s}^{n}\left(t_{\Lambda}\right)} \frac{f_{1}^{(n)}\left(x_{1}^{(n)}, t_{X}\right)}{f_{1}^{(n)}\left(x_{1}^{(n)}, t_{\Lambda}\right)} \frac{f_{2}^{(n)}\left(x_{2}^{(n)}, t_{X}\right)}{f_{2}^{(n)}\left(x_{2}^{(n)}, t_{\Lambda}\right)},
$$

where $E_{T}(i)$ is the transverse energy $E_{T}$ of a parton $i$ in the $\{p\}_{X+n}$ and $t_{X}$ is defined by

$$
t_{X}^{2}=E_{T}^{2}(t)+E_{T}^{2}(\bar{t})+\sum_{i=1}^{n} E_{T}^{2}(i)
$$


Once the PDF and $\alpha_{s}$ weight is calculated, the event sample is re-weighted. However, since the weight is not bounded above by unity, the upper bound of the weight must be found at first by calculating the weight for a large number of the events $\{p\}_{X+n}$. The integrated LO cross section obtained in the step 1 has to be multiplied by the obtained upper bound of the weight.

5. A parton shower evolution is performed on the event sample, and the exclusive $n$ jet event is produced when $n<n_{\max }$ or the inclusive $n$-jet event is produced when $n=n_{\max }$, by using the method described in this section. The parameter $C_{\text {match }}$ is set to a constant value $C_{\text {match }}=1.5$. The parton shower starting scale, which is the maximal shower evolution scale, is determined based on the information of the generated event $\{p\}_{X+n}$ (independently of the shower history) and is defined by a square root of

$$
\frac{1}{6}\left\{E_{T}^{2}(t)+E_{T}^{2}(\bar{t})+\sum_{i=1}^{n} E_{T}^{2}(i)\right\}
$$

The overall factor $1 / 6$ is chosen so that the total cross section after merging is not deviated so much from the LO inclusive cross section $\sigma(p p \rightarrow X)$ (the total cross section will be given in table 1 ).

6. Repeat the above procedure from the step 2 to the step 5 until a large number of the accepted event samples are obtained.

\section{$3 \quad$ Numerical studies}

In this section, the new merging algorithm described in section 2 is numerically studied in detail. I call it the modelA algorithm in this paper. The top quark pair production process at the $14 \mathrm{TeV}$ LHC is simulated. The results are compared against those of other existing merging algorithms, the CKKW-L $[9,12,20]$ and the MLM $[13,14]$ algorithms.

\subsection{Setup and the cross section}

In my implementation of the CKKW-L algorithm, ${ }^{1}$ the PDF and $\alpha_{s}$ weight is calculated in the same way as the steps 3 and 4 of the merging procedure described in section 2 . The Sudakov form factors are calculated by using the Sudakov veto algorithm [9] based on the information of the constructed parton shower history. If the shower history is not constructed (see the step 3 of the merging procedure), the intermediate Sudakov form factors are set unity and instead the PDF and $\alpha_{s}$ weight takes the following form which is smaller than eq. (2.12):

$$
\frac{\alpha_{s}^{2+n}\left(t_{X}\right)}{\alpha_{s}^{2+n}\left(t_{\Lambda}\right)} \frac{f_{1}^{(n)}\left(x_{1}^{(n)}, t_{X}\right)}{f_{1}^{(n)}\left(x_{1}^{(n)}, t_{\Lambda}\right)} \frac{f_{2}^{(n)}\left(x_{2}^{(n)}, t_{X}\right)}{f_{2}^{(n)}\left(x_{2}^{(n)}, t_{\Lambda}\right)} .
$$

\footnotetext{
${ }^{1}$ The method of phase space separation is slightly different from the original one in my implementation. This is the one called the CKKW-L+ algorithm in ref. [26]. However, numerical differences are found small. The difference appears as the different values of the cross section $\sigma(t \bar{t}+1)$ for $n_{\max }=2$ and 3 shown in the third and fourth rows of table 1.
} 


\begin{tabular}{|c|c|c|c|c||c|}
\hline & $\sigma(t \bar{t}+0)(\mathrm{pb})$ & $\sigma(t \bar{t}+1)(\mathrm{pb})$ & $\sigma(t \bar{t}+2)(\mathrm{pb})$ & $\sigma(t \bar{t}+3)(\mathrm{pb})$ & Total (pb) \\
\hline ModelA_2 & 240 & 155 & 118 & - & 513 \\
\hline ModelA_3 & 240 & 155 & 68 & 43 & 506 \\
\hline CKKWL_2 & 133 & 160 & 232 & - & 525 \\
\hline CKKWL_3 & 133 & 159 & 109 & 97 & 498 \\
\hline MLM_2 & 230 & 153 & 124 & - & 507 \\
\hline MLM_3 & 230 & 153 & 69 & 46 & 498 \\
\hline
\end{tabular}

Table 1. The contribution to the total cross section from each of the LO $t \bar{t}+0,1,2,3$-parton production cross sections and the sum of them in unit of pico barn (pb). The index after the name of the merging algorithm means $n_{\max }$. The LO inclusive cross section is $496 \mathrm{pb}$.

The definitions of the several variables in the above weight are given in the steps 3 and 4 of the merging procedure in section 2 and $t_{X}$ is defined by eq. (2.13). In my implementation of the MLM algorithm, the PDF and $\alpha_{s}$ weight is calculated exactly in the same way as the modelA algorithm and the parton shower starting scale is also set in the same way (eq. (2.14)). When the parton shower history cannot be constructed, the PDF and $\alpha_{s}$ weight in the CKKW-L algorithm (eq. (3.1)) is different from that in the other two algorithms (eq. (2.12)). However, I have found that the ratio of the shower history nonconstructed events divided by the total events is negligibly small: $0 \%$ in the $t \bar{t}+1$-parton process, $0.04 \%$ in the $t \bar{t}+2$-parton process and $0.09 \%$ in the $t \bar{t}+3$-parton process. Therefore, with my implementations of the three merging algorithms, the difference among the three algorithms is only how the Sudakov suppression is calculated. Note that the parameter $C_{\text {match }}$ also takes the same value

$$
C_{\text {match }}=1.5
$$

in the modelA and the MLM algorithms.

The generation cutoff $Q_{\mathrm{cut}}^{\mathrm{ME}}$ in eq. (2.1) is set the same in all of the three algorithms:

$$
R_{\text {cut }}^{\mathrm{ME}}=0.4, \quad p_{T \text { cut }}^{\mathrm{ME}}=20 \mathrm{GeV} .
$$

The rapidity cutoffs $y_{\text {cut }}^{\text {clus }}$ and $y_{\text {cut }}^{\text {detect }}$ on final state partons for the jet clustering, which are defined above eqs. (2.4) and (2.5) respectively, are set to

$$
y_{\text {cut }}^{\text {clus }}=y_{\text {cut }}^{\text {detect }}=5 \text {, }
$$

and jets are defined by the anti- $k_{T}$ algorithm with the parameters

$$
R^{\text {jet }}=0.4, \quad p_{T \text { cut }}^{\text {jet }}=30 \mathrm{GeV} .
$$

The rapidity cutoff on jets is set to

$$
y_{\text {cut }}^{\text {jet }}=4.8 \text {. }
$$

The merging scales in the three algorithms are set as follows: 
- ModelA $: R^{\mathrm{MS}}=0.4, \quad p_{T \text { cut }}^{\mathrm{MS}}=30 \mathrm{GeV}$

- CKKW-L : $R^{\mathrm{MS}}=0.4, \quad p_{T \text { cut }}^{\mathrm{MS}}=20 \mathrm{GeV}$

- MLM $\quad: R^{\mathrm{MS}}=0.4, \quad E_{T \text { cut }}^{\mathrm{MS}}=30 \mathrm{GeV}$

The merging scale in the modelA algorithm is the same as the parameters of the anti- $k_{T}$ algorithm defined in eq. (3.5) by definition. The CKKW-L algorithm allows us to set the merging scale equal to the generation cutoff. In the cone jet algorithm used in the MLM algorithm, a cutoff on jets is given not by the transverse momentum $p_{T}$ but by the transverse energy $E_{T}$. Since $E_{T} \geq p_{T}$ is always true, the merging scale choice in the MLM algorithm corresponds to a smaller merging scale than that in the modelA algorithm.

The event samples for the $t \bar{t}+0,1,2,3$-parton production processes at proton-proton (pp) collisions are generated according to the leading order (LO) cross sections by using MadGraph5_aMC@NLO [38] version 5.2.2.1. For the PDFs set, CTEQ6L1 [39] is used. PYTHIA8 [30, 31] version 8212 is used for the parton shower evolution. Fastjet [40] version 3.1.0 is used for executing the anti- $k_{T}$ algorithm. The cone jet clustering algorithm in the MLM algorithm is executed by using the CellJet routine implemented in PYTHIA8. The $t \bar{t}$ is assumed stable, since the main purpose of this study is to investigate a way of accurately modelling the kinematic activity of jets induced by the hard process. Hence the hadronisation after the shower evolution and the multiple interaction in PYTHIA8 are also turned off. The $t \bar{t}$ will not be clustered to construct jets.

In table 1 , the contribution to the total cross section from each of the leading order (LO) $t \bar{t}+0,1,2,3$-parton production cross sections and the sum of them in unit of pico barn $(\mathrm{pb})$ are summarised. The index after the name of the merging algorithm represents the maximal number of partons produced by the LO cross section, $n_{\max }$. This notation is used hereafter. It is shown that the modelA results reasonably reproduce the LO inclusive cross section, which is $496 \mathrm{pb}$. The $t \bar{t}+2,3$-parton cross sections in the CKKWL results are particularly larger than those in the other results, and those in the MLM results are slightly larger than those in the modelA results. These observations reflect the fact that the smaller the merging scale is, the larger the contribution from a cross section for higher parton multiplicity will be.

\subsection{Distributions}

In figure 1, I show the normalised distributions of $k_{T}$ scales [32] $d_{i}$ associated with merging from $i+1$ to $i$ jets for $i=1,2,3$ and the scalar sum $H_{T}$ of the $p_{T}$ of the $t \bar{t}$ and all jets. In all of the panels, the solid curves represent the modelA_3 results, the dashed curves represent the modelA $\_2$ results and $\times, \square, \diamond$ and $\nabla$ points represent the CKKWL_3, CKKWL_2, MLM_3 and MLM_2 results, respectively. The ratios with respect to the modelA_3 results are shown in the lower part of each panel. When the different algorithms are compared, the algorithms with the same $n_{\max }$ should be compared, to be fair. It is shown that the modelA results agree very well with the MLM results. The agreement between the modelA results and the CKKWL results is also reasonably good as a whole. However, the CKKWL results slightly tend to show harder distributions. We can confirm the validity of the modelA 

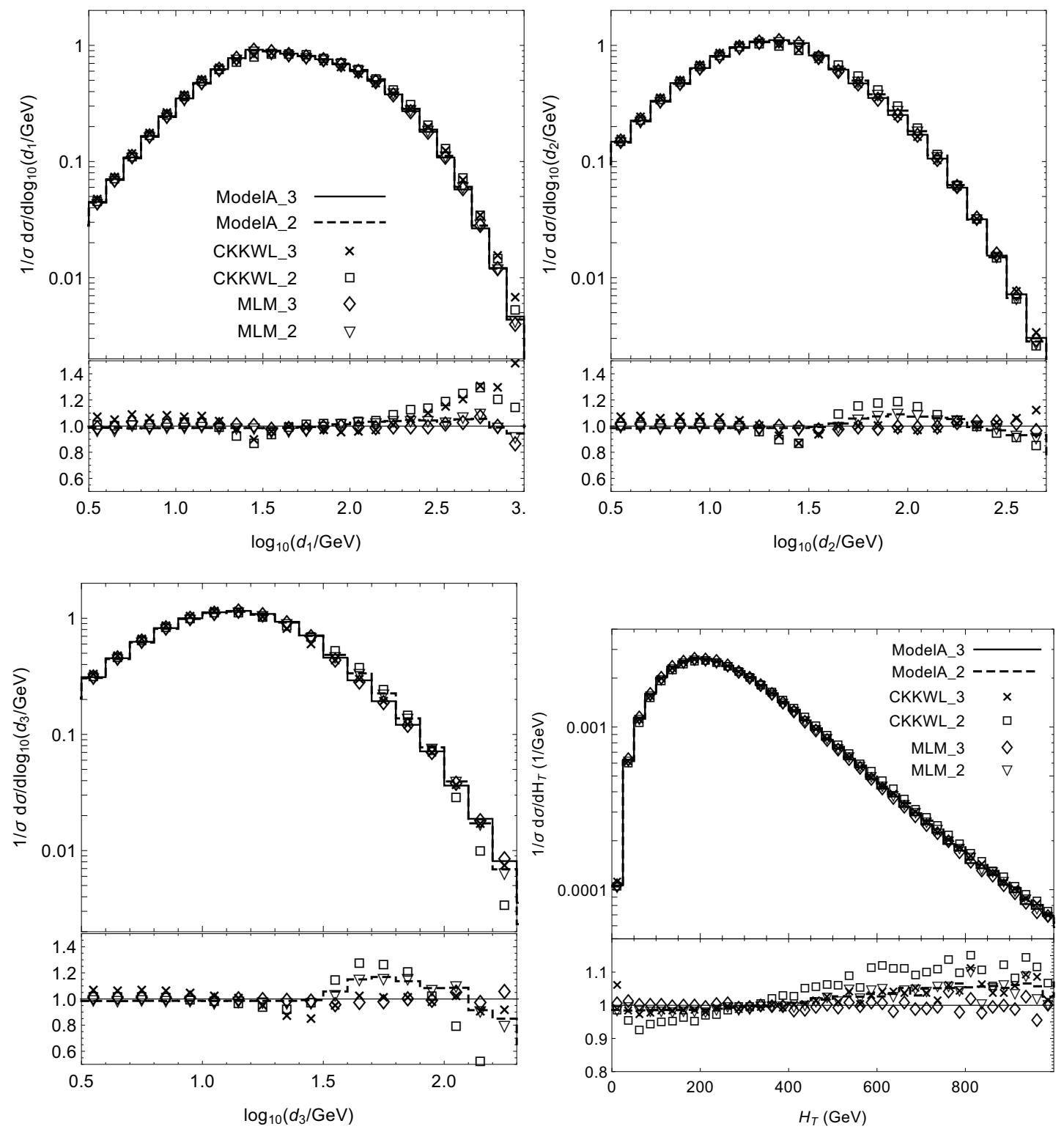

Figure 1. The normalised distributions of $k_{T}$ scales $d_{i}$ associated with merging from $i+1$ to $i$ jets for $i=1,2,3$ and the scalar sum $H_{T}$ of the $p_{T}$ of the $t \bar{t}$ and all jets. The solid curves give the modelA $\_3$ results, the dashed curves give the modelA_2 results and $\times, \square, \diamond$ and $\nabla$ points give the CKKWL_3, CKKWL_2, MLM_3 and MLM_2 results, respectively. The ratios with respect to the modelA_3 results are shown in the lower part of each panel. The index after the name of the merging algorithm means the maximal number of partons produced by the LO cross section, $n_{\max }$.

algorithm as a merging algorithm from the good agreement with the other results and the smooth distributions of the modelA results.

Not only the fully inclusive events are produced, but also the behaviour of jets must be described appropriately by a merging algorithm. In figure 2, I show the normalised 
differential cross sections as functions of the $p_{T}$ and rapidity $y$ of the highest $p_{T}$ jet and those of the second highest $p_{T}$ jet in the inclusive two-jet events $\left(n_{\text {jets }} \geq 2\right.$, where $n_{\text {jets }}$ denotes the number of jets). The correspondence between the curves/points and the merging methods is shown inside the upper left panel and it is the same as in figure 1. The ratios with respect to the modelA_3 results are shown in the lower part of each panel. The modelA results again show good agreement with the MLM results in all of the observables. When the three algorithms are compared for $n_{\max }=3$, the CKKWL result shows harder $p_{T}$ spectra and broader $y$ spectra than the other two results. This is consistent with the observation in ref. [14], where the CKKW-L algorithm implemented in Ariadne and the CKKW algorithm implemented in SHERPA tend to show harder $p_{T}$ and broader pseudo-rapidity spectra of jets than the MLM algorithms implemented in Alpgen and Helac and the $k_{T^{-}}$jet MLM algorithm implemented in MadGraph. In my implementations of the three algorithms, the PDF and $\alpha_{s}$ weight is calculated in a common way and the matrix-element generator and the parton shower generator are also the same. Therefore, it can be concluded that these discrepancies in the $p_{T}$ and $y$ spectra originate from the different ways of calculating the Sudakov suppression.

As an observable that measures angular correlations among jets, I choose the azimuthal angle difference $[6,7]$ between the two highest $p_{T}$ jets:

$$
\Delta \phi=\phi_{1}-\phi_{2}
$$

The following rapidity cuts are imposed on the two highest $p_{T}$ jets

$$
y_{2}<0<y_{1}, \quad \Delta y=y_{1}-y_{2}>4,
$$

which are often called the vector boson fusion $(\mathrm{VBF})$ cuts and enhance the correlation. One of the two jets which has a positive rapidity $y_{1}$ is chosen for the $\phi_{1}$ and the other jet which has a negative rapidity $y_{2}$ is chosen for the $\phi_{2}$. In order to enhance the correlation in $\Delta \phi$ further, the following cut is imposed on the $t \bar{t}$ invariant mass [7],

$$
m_{t \bar{t}}<600 \mathrm{GeV}
$$

Before I show the $\Delta \phi$ distributions, I study the minimum $\Delta R=\sqrt{\Delta y^{2}+\Delta \phi^{2}}$ between the highest $p_{T}$ jet and the matrix-element parton, labelled as $\Delta R_{1}$, and that between the second highest $p_{T}$ jet and the matrix-element parton, labelled as $\Delta R_{2}$. A large value in $\Delta R_{1}$ or $\Delta R_{2}$ implies that the corresponding jet loses the kinematic information of the matrix-element parton. Therefore, large values in either or both of $\Delta R_{1,2}$ imply the loss of the LO accuracy of the angular correlation in $\Delta \phi . \Delta R_{1,2}$ are calculated according to the following procedure:

1. Pick up an event sample which contains at least two jets and further satisfies the cuts in eqs. (3.8) and (3.9).

2. Pick up the highest $p_{T}$ jet from the two highest $p_{T}$ jets and calculate $\Delta R$ between the jet and each of the $n$ partons produced by the LO $t \bar{t}+n$-parton cross section. The minimum value of $\Delta R$ becomes $\Delta R_{1}$. 

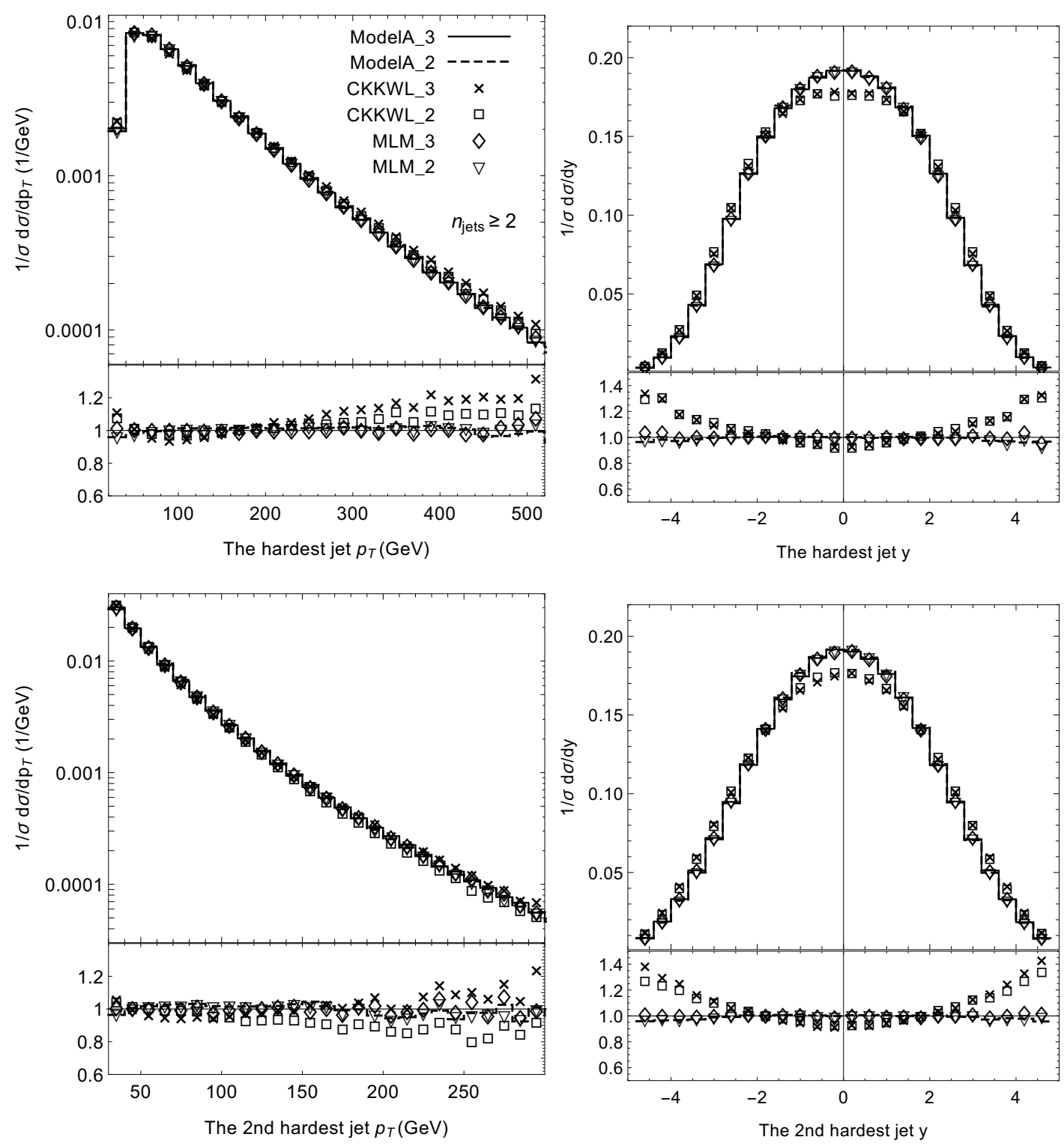

Figure 2. The normalised differential cross sections as functions of the $p_{T}$ and $y$ of the highest $p_{T}$ jet and those of the second highest $p_{T}$ jet in the inclusive two-jet events. The correspondence between the curves/points and the merging methods is shown inside the left upper panel and it is the same as in figure 1. The ratios with respect to the modelA_3 results are shown in the lower part of each panel.

3. Pick up the second highest $p_{T}$ jet from the two highest $p_{T}$ jets and calculate $\Delta R$ between the jet and each of the $n$ partons produced by the LO $t \bar{t}+n$-parton cross section. The parton which gives the $\Delta R_{1}$ in the step 2 will not be considered. The minimum value of $\Delta R$ becomes $\Delta R_{2}$.

Figure 3 shows the normalised differential cross sections as functions of $\Delta R_{1}$ and $\Delta R_{2}$ in the exclusive two-jet events (upper panels) and the inclusive two-jet events (lower panels). 
The correspondence between the curves/points and the merging methods is shown inside the upper left panel and it is the same as in figure 1. The ratios with respect to the CKKWL $\_2$ results are shown in the lower part of each panel (Note that this is different from the other figures. The reason is because the modelA_3 results give 0 in $\Delta R_{1,2}$ ). The last bins in the $\Delta R_{1,2}$ distributions contain all of the events which give $\Delta R_{1,2}>1$. When the two-jet events originate from either of the LO $t \bar{t}+0,1$-parton cross sections, the angular correlation between the two jets is obviously not at the LO accuracy. In table 2, I show the ratios of the contributions to the exclusive two-jet events from each of the LO $t \bar{t}+0,1,2,3$-parton production cross sections divided by the sum of them in unit of $\%$. The events satisfy the cuts in eqs. (3.8) and (3.9). While the contributions from the LO $t \bar{t}+0,1$-parton cross sections are zero in the modelA results, they are non-zero in the other results. Note that the non-zero contribution from the LO $t \bar{t}+3$-parton cross section in the modelA_3 result is due to the fact that one of the three jets is removed by the rapidity cut in eq. (3.6). When the event originates from the LO $t \bar{t}+0$-parton cross section, both of $\Delta R_{1,2}$ cannot be calculated and so the event will be contained in the last bins in the $\Delta R_{1,2}$ distributions. When the event originates from the LO $t \bar{t}+1$-parton cross section, $\Delta R_{2}$ cannot be calculated and so the event will be contained in the last bin in the $\Delta R_{2}$ distribution.

In figure 3 , the modelA results always give the sharp cutoff at $\Delta R_{1,2}=0.6$ and zero above this, as expected from the construction of the modelA algorithm (eq. (2.4)) and eqs. (3.2) and (3.5). In the exclusive two-jet events (upper two panels), the modelA_2 result and the modelA_3 result give the same distribution, since only the LO $t \bar{t}+2$-parton cross section contributes in the both cases (see table 2). The MLM results show differences from the modelA results around $\Delta R_{1,2}=0.6$ due to the difference between the cone jet algorithm used during the merging procedure and the anti- $k_{T}$ jet algorithm used to define the jets in this figure. The MLM results give the visible contributions in the last bins, while the modelA results do not. However, as a whole, the MLM results are very similar to the modelA results. Since $\Delta R_{1,2}$ are not physical observables, the sharp cutoffs in their distributions will not indicate the invalidity of the modelA and the MLM algorithms. The $\Delta R_{1}$ distribution in the $n_{\text {jets }}=2$ events (upper left panel) shows that the CKKWL $\_3$ result is the best in the sense that it has more events with small $\Delta R_{1}$ than the other results. The other results are comparable with each other in the statistically dominant region $\left(\Delta R_{1} \simeq 0.0 \sim 0.3\right)$. The $\Delta R_{2}$ distribution in the $n_{\text {jets }}=2$ events (upper right panel) shows that the CKKWL $\_2$ result is the worst in the sense that it has more events with large $\Delta R_{2}$ than the other results. The other results are comparable with each other in the statistically dominant region $\left(\Delta R_{2} \simeq 0.0 \sim 0.3\right)$. As mentioned above, large values in either or both of $\Delta R_{1}$ and $\Delta R_{2}$ imply the loss of the LO accuracy of the angular correlation in $\Delta \phi$. Therefore, the upper two panels in figure 3 imply that the CKKWL 22 result contains more events which lose the LO accuracy of the angular correlation in $\Delta \phi$ than the other results. The similar behaviour is observed in the $n_{\text {jets }} \geq 2$ events (lower panels).

In figure 4, I show the normalised differential cross sections as functions of $|\Delta \phi|$. The results in the exclusive two-jet events are shown in the upper left panel and those in the inclusive two-jets events are shown in the upper right panel. Furthermore, the results with 

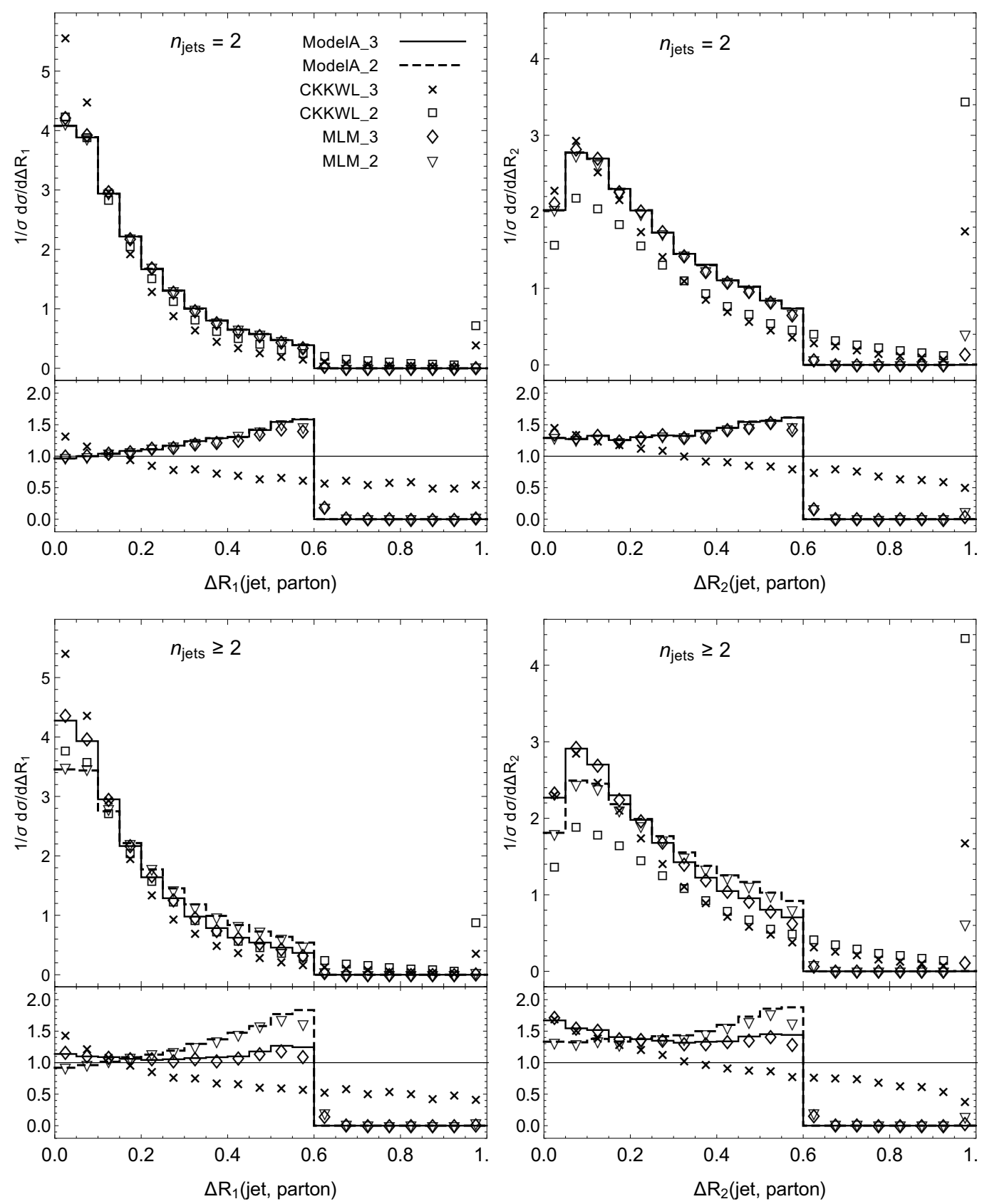

Figure 3. The minimum $\Delta R=\sqrt{\Delta y^{2}+\Delta \phi^{2}}$ between the highest $p_{T}$ jet and the matrix-element parton (labelled as $\Delta R_{1}$ ) and that between the second highest $p_{T}$ jet and the matrix-element parton (labelled as $\Delta R_{2}$ ), in the exclusive (upper panels) and inclusive (lower panels) two-jet events. The cuts in eqs. (3.8) and (3.9) are imposed. The correspondence between the curves/points and the merging methods is shown inside the upper left panel and it is the same as in figure 1 . The ratios with respect to the CKKWL_2 results are shown in the lower part of each panel. The last bins contain all of the events which give $\Delta R_{1,2}>1$. 


\begin{tabular}{|c|c|c|c|c|}
\hline & $\sigma(t \bar{t}+0) /$ Total & $\sigma(t \bar{t}+1) /$ Total & $\sigma(t \bar{t}+2) /$ Total & $\sigma(t \bar{t}+3) /$ Total \\
\hline ModelA_2 & 0 & 0 & 100 & - \\
\hline ModelA_3 & 0 & 0 & 99.9 & 0.1 \\
\hline CKKWL_2 & 0.6 & 4.3 & 95.1 & - \\
\hline CKKWL_3 & 0.7 & 4.5 & 47.2 & 47.7 \\
\hline MLM_2 & $<0.05$ & 0.6 & 99.4 & - \\
\hline MLM_3 & $<0.05$ & 0.7 & 93.3 & 6.0 \\
\hline
\end{tabular}

Table 2. The ratios of the contributions to the exclusive two-jet events from each of the LO $t \bar{t}+0,1,2,3$-parton production cross sections divided by the sum of them in unit of \%. The cuts in eqs. (3.8) and (3.9) are imposed.

a different rapidity cut $\Delta y=y_{1}-y_{2}>3$ instead of $\Delta y>4$ in eq. (3.8) are shown in the lower two panels. The correspondence between the curves/points and the merging methods is shown inside the upper left panel and it is the same as in figure 1. The ratios with respect to the modelA_3 results are shown in the lower part of each panel. It is shown that all the results agree with each other in $\pm 10 \%$. This observation might be surprising considering the differences found in figures 2 and 3. By looking carefully at the results, we can observe that the CKKWL_2 results consistently show the visible deviations from the other results near the bottom region $(|\Delta \phi| \sim 0)$ and the peak region $(|\Delta \phi| \sim 2)$ in all of the panels. Since the CKKWL_2 results are deviated in a way that their distributions become flatter than the other results, it can be concluded that these deviations are induced by the loss of the LO accuracy of the angular correlation in $\Delta \phi$ which has been already implied by the large $\Delta R_{2}$ in figure 3. The differences between the modelA_2 results and the modelA_3 results in the $n_{\text {jets }} \geq 2$ events are small. This is as expected from the construction of the modelA algorithm that the LO accuracy of the angular correlation between the two highest $p_{T}$ jets is preserved even in the inclusive two-jet events in the modelA $\_2$ result. The differences between the modelA results and the MLM results are found small.

\section{Summary and discussion}

In this paper, a new tree level merging algorithm which guarantees the leading order (LO) accuracy of angular correlations among jets is proposed. The new algorithm proceeds based on the same philosophy of the MLM algorithm and the following idea is additionally implemented: the definition of jets used during a merging procedure is set identical to the one used during analyses of jets. The new algorithm allows us to produce fully inclusive event samples as other existing merging algorithms. In inclusive event samples, the kinematics of each of up to the $n_{\max }$ highest transverse momentum $p_{T}$ jets strictly follows that of each of the matrix-element partons, where $n_{\max }$ denotes the maximal number of partons produced by a LO cross section. Therefore, as long as the highest $p_{T}$ jets are always picked up, the LO accuracy of angular correlations among up to $n_{\max }$ jets is preserved.

The detailed merging procedure of the new merging algorithm (named modelA in this paper) is explained in the case that the anti- $k_{T}$ algorithm is chosen as a jet clustering algorithm used during the merging procedure and at the analysis level. The validation 

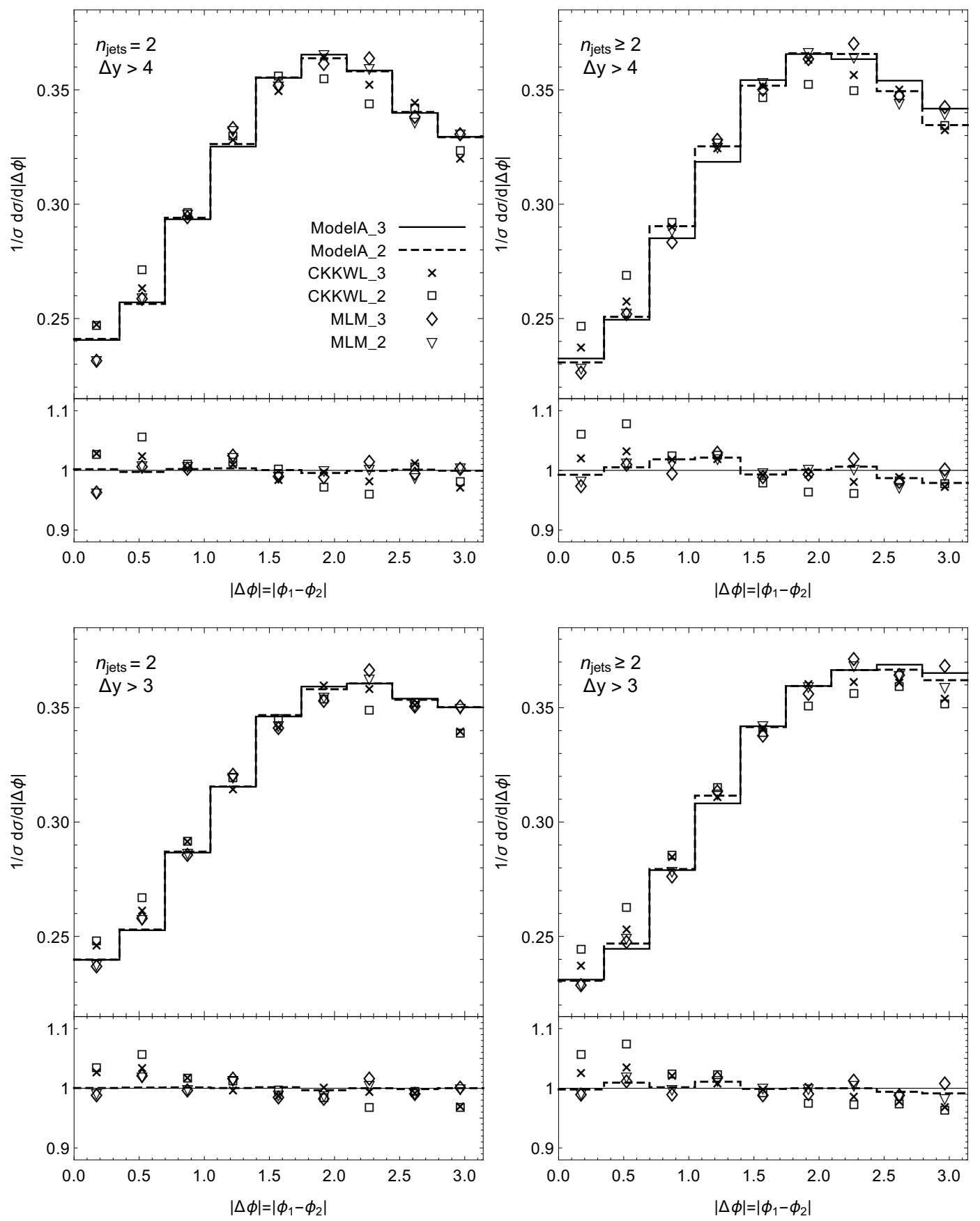

Figure 4. The normalised differential cross sections as functions of $|\Delta \phi|=\left|\phi_{1}-\phi_{2}\right|$ in the $n_{\text {jets }}=2$ events (left panels) and the $n_{\text {jets }} \geq 2$ events (right panels). The cuts in eqs. (3.8) and (3.9) are imposed in the upper two panels. A different rapidity cut $\Delta y>3$ instead of $\Delta y>4$ is imposed in the lower two panels. The correspondence between the curves/points and the merging methods is shown inside the upper left panel and it is the same as in figure 1 . The ratios with respect to the modelA_3 results are shown in the lower part of each panel. 
test is also performed in this case (summarised below). However, the modelA algorithm can be valid whatever the definition of jets is. The generation cutoff and the matching measure corresponding to an arbitrary jet clustering algorithm can be easily read from the measure of the jet clustering algorithm. I have explicitly presented those corresponding to the inclusive/exclusive $k_{T}$ algorithms and the inclusive $p p$ Cambridge/Aachen algorithm.

The $k_{T}$ scales $d_{i}$ associated with merging from $i+1$ to $i$ jets for $i=1,2,3$ and the scalar sum $H_{T}$ of the $p_{T}$ of the top quark, the anti-top quark and all jets in the inclusive $t \bar{t}$ events are produced. Furthermore, the $p_{T}$ and the rapidity $y$ of the two highest $p_{T}$ jets in the inclusive $t \bar{t}$ plus two-jet events are studied. The results are compared with those of the CKKW-L algorithm and the MLM algorithm. The results agree very well with the MLM results. This observation confirms the validity of the modelA algorithm as a merging algorithm, when jets are defined by the anti- $k_{T}$ algorithm. Compared to the CKKW-L results, the modelA results (thus the MLM results, too) slightly tend to show softer $p_{T}$ and narrower $y$ spectra of jets. This is consistent with the observation in ref. [14]. Since the $\mathrm{PDF}$ and $\alpha_{s}$ weight is calculated in a common way and the matrix-element generator and the parton shower generator are also the same in my implementations of the three merging algorithms, I have concluded that these discrepancies originate from the different ways of calculating the Sudakov suppression.

$\Delta R_{1}$ (the minimum $\Delta R=\sqrt{\Delta y^{2}+\Delta \phi^{2}}$ between the highest $p_{T}$ jet and the matrixelement parton) and $\Delta R_{2}$ (the minimum $\Delta R$ between the second highest $p_{T}$ jet and the matrix-element parton) are found interesting quantities, since large values in either or both of $\Delta R_{1,2}$ imply the loss of the LO accuracy of the angular correlation between the two highest $p_{T}$ jets. Although the MLM results give some events which have large $\Delta R_{1,2}$, the MLM results have quite similar spectra with the modelA results. In $\Delta R_{1}$, the CKKWL_3 results (the index indicates $n_{\max }$ ) are the best (more events in smaller $\Delta R_{1}$ ), while the other results are comparable with each other in statistically dominant region. In $\Delta R_{2}$, the CKKWL $\_2$ results are the worst (more events in larger $\Delta R_{2}$ ), while the other results are comparable with each other in statistically dominant region.

As an angular correlation observable, the azimuthal angle difference $\Delta \phi=\phi_{1}-\phi_{2}$ between the two highest $p_{T}$ jets is studied. The vector boson fusion cuts are imposed on the jets. The results of all the three merging algorithms agree with each other in $\pm 10 \%$. However, by looking carefully, it is observed that the CKKWL_2 results consistently show the visible deviations from the other results near the bottom region $(|\Delta \phi| \sim 0)$ and the peak region $(|\Delta \phi| \sim 2)$. The CKKWL 22 results are deviated in a way that their distributions become flatter than the other results. Therefore, I have concluded that the deviations are induced by the loss of the LO accuracy of the angular correlation, which has been already implied by the large $\Delta R_{2}$ in the CKKWL_2 results.

The differences between the modelA results and the MLM results are found small not only in the $p_{T}$ and $y$ but also in the angular correlation observable $\Delta \phi$, when jets are defined by the anti- $k_{T}$ algorithm. However, it has been shown that the MLM results contain some event samples which have large $\Delta R_{1,2}$. Furthermore, if jets at the analysis level are defined by other algorithms, the MLM results might contain the such event sample more and the differences between the modelA result and the MLM result in angular correlation 
observables might become larger. Therefore, it will be safer to use the modelA algorithm for jet angular correlation studies. An implementation of the modelA algorithm is as simple as that of the MLM algorithm.

There are basically two parameters which cannot be determined uniquely in the new algorithm, $C_{\text {match }}$ and the definition of the parton shower starting scale (this is also the case in the MLM algorithm). This fact might be seen as a weak point in the algorithm. However, merging algorithms and parton shower programs are just models after all. An appropriate approach may be to tune the algorithm together with a parton shower model by using these parameters so that the $p_{T}$ and $y$ distributions of jets in a given process become consistent with the data at first and then make the predictions of angular correlations among the jets.

\section{Acknowledgments}

The work of J.N. is supported by the Institutional Strategy of the University of Tübingen (DFG, ZUK 63).

Open Access. This article is distributed under the terms of the Creative Commons Attribution License (CC-BY 4.0), which permits any use, distribution and reproduction in any medium, provided the original author(s) and source are credited.

\section{References}

[1] T. Plehn, D.L. Rainwater and D. Zeppenfeld, Determining the structure of Higgs couplings at the LHC, Phys. Rev. Lett. 88 (2002) 051801 [hep-ph/0105325] [INSPIRE].

[2] V. Del Duca, W. Kilgore, C. Oleari, C. Schmidt and D. Zeppenfeld, Gluon fusion contributions to $H+2$ jet production, Nucl. Phys. B 616 (2001) 367 [hep-ph/0108030] [INSPIRE].

[3] V. Hankele, G. Klamke, D. Zeppenfeld and T. Figy, Anomalous Higgs boson couplings in vector boson fusion at the CERN LHC, Phys. Rev. D 74 (2006) 095001 [hep-ph/0609075] [INSPIRE].

[4] G. Klamke and D. Zeppenfeld, Higgs plus two jet production via gluon fusion as a signal at the CERN LHC, JHEP 04 (2007) 052 [hep-ph/0703202] [INSPIRE].

[5] K. Hagiwara, Q. Li and K. Mawatari, Jet angular correlation in vector-boson fusion processes at hadron colliders, JHEP 07 (2009) 101 [arXiv: 0905.4314] [INSPIRE].

[6] M.R. Buckley and M.J. Ramsey-Musolf, Diagnosing Spin at the LHC via Vector Boson Fusion, JHEP 09 (2011) 094 [arXiv: 1008.5151] [INSPIRE].

[7] K. Hagiwara and S. Mukhopadhyay, Azimuthal correlation among jets produced in association with a bottom or top quark pair at the LHC, JHEP 05 (2013) 019 [arXiv: 1302.0960] [INSPIRE].

[8] S. Catani, F. Krauss, R. Kuhn and B.R. Webber, QCD matrix elements + parton showers, JHEP 11 (2001) 063 [hep-ph/0109231] [INSPIRE].

[9] L. Lönnblad, Correcting the color dipole cascade model with fixed order matrix elements, JHEP 05 (2002) 046 [hep-ph/0112284] [INSPIRE]. 
[10] F. Krauss, Matrix elements and parton showers in hadronic interactions, JHEP 08 (2002) 015 [hep-ph/0205283] [INSPIRE].

[11] S. Mrenna and P. Richardson, Matching matrix elements and parton showers with HERWIG and PYTHIA, JHEP 05 (2004) 040 [hep-ph/0312274] [INSPIRE].

[12] N. Lavesson and L. Lönnblad, W+jets matrix elements and the dipole cascade, JHEP $\mathbf{0 7}$ (2005) 054 [hep-ph/0503293] [INSPIRE].

[13] M.L. Mangano, M. Moretti, F. Piccinini and M. Treccani, Matching matrix elements and shower evolution for top-quark production in hadronic collisions, JHEP 01 (2007) 013 [hep-ph/0611129] [INSPIRE].

[14] J. Alwall et al., Comparative study of various algorithms for the merging of parton showers and matrix elements in hadronic collisions, Eur. Phys. J. C 53 (2008) 473 [arXiv: 0706.2569] [INSPIRE].

[15] W.T. Giele, D.A. Kosower and P.Z. Skands, A simple shower and matching algorithm, Phys. Rev. D 78 (2008) 014026 [arXiv:0707.3652] [INSPIRE].

[16] J. Alwall, S. de Visscher and F. Maltoni, QCD radiation in the production of heavy colored particles at the LHC, JHEP 02 (2009) 017 [arXiv: 0810.5350] [INSPIRE].

[17] S. Hoeche, F. Krauss, S. Schumann and F. Siegert, QCD matrix elements and truncated showers, JHEP 05 (2009) 053 [arXiv: 0903.1219] [INSPIRE].

[18] K. Hamilton, P. Richardson and J. Tully, A Modified CKKW matrix element merging approach to angular-ordered parton showers, JHEP 11 (2009) 038 [arXiv:0905.3072] [INSPIRE].

[19] W.T. Giele, D.A. Kosower and P.Z. Skands, Higher-Order Corrections to Timelike Jets, Phys. Rev. D 84 (2011) 054003 [arXiv:1102.2126] [INSPIRE].

[20] L. Lönnblad and S. Prestel, Matching Tree-Level Matrix Elements with Interleaved Showers, JHEP 03 (2012) 019 [arXiv:1109.4829] [INSPIRE].

[21] L. Lönnblad and S. Prestel, Unitarising Matrix Element + Parton Shower merging, JHEP 02 (2013) 094 [arXiv: 1211.4827] [INSPIRE].

[22] S. Plätzer, Controlling inclusive cross sections in parton shower + matrix element merging, JHEP 08 (2013) 114 [arXiv:1211.5467] [INSPIRE].

[23] V.N. Gribov and L.N. Lipatov, Deep inelastic e p scattering in perturbation theory, Sov. J. Nucl. Phys. 15 (1972) 438 [InSPIRE].

[24] G. Altarelli and G. Parisi, Asymptotic Freedom in Parton Language, Nucl. Phys. B 126 (1977) 298 [INSPIRE].

[25] Y.L. Dokshitzer, Calculation of the Structure Functions for Deep Inelastic Scattering and $e^{+} e^{-}$Annihilation by Perturbation Theory in Quantum Chromodynamics., Sov. Phys. JETP 46 (1977) 641 [INSPIRE].

[26] K. Hagiwara and J. Nakamura, Study on the azimuthal angle correlation between two jets in the top quark pair production, arXiv:1501.00794 [INSPIRE].

[27] T. Sjöstrand and P.Z. Skands, Transverse-momentum-ordered showers and interleaved multiple interactions, Eur. Phys. J. C 39 (2005) 129 [hep-ph/0408302] [InSPIRE].

[28] R. Corke and T. Sjöstrand, Interleaved Parton Showers and Tuning Prospects, JHEP 03 (2011) 032 [arXiv:1011.1759] [INSPIRE]. 
[29] E. Norrbin and T. Sjöstrand, QCD radiation off heavy particles, Nucl. Phys. B 603 (2001) 297 [hep-ph/0010012] [INSPIRE].

[30] T. Sjöstrand, S. Mrenna and P.Z. Skands, A Brief Introduction to PYTHIA 8.1, Comput. Phys. Commun. 178 (2008) 852 [arXiv:0710.3820] [INSPIRE].

[31] T. Sjöstrand, S. Mrenna and P.Z. Skands, PYTHIA 6.4 Physics and Manual, JHEP 05 (2006) 026 [hep-ph/0603175] [INSPIRE].

[32] S. Catani, Y.L. Dokshitzer, M.H. Seymour and B.R. Webber, Longitudinally invariant $K_{t}$ clustering algorithms for hadron hadron collisions, Nucl. Phys. B 406 (1993) 187 [InSPIRE].

[33] M. Cacciari, G.P. Salam and G. Soyez, The Anti-k(t) jet clustering algorithm, JHEP 04 (2008) 063 [arXiv:0802.1189] [INSPIRE].

[34] S.D. Ellis and D.E. Soper, Successive combination jet algorithm for hadron collisions, Phys. Rev. D 48 (1993) 3160 [hep-ph/9305266] [INSPIRE].

[35] Y.L. Dokshitzer, G.D. Leder, S. Moretti and B.R. Webber, Better jet clustering algorithms, JHEP 08 (1997) 001 [hep-ph/9707323] [INSPIRE].

[36] M. Wobisch and T. Wengler, Hadronization corrections to jet cross-sections in deep inelastic scattering, hep-ph/9907280 [INSPIRE].

[37] J. Alwall and S. de Visscher, Introduction to jet-parton matching in $M G / M E$, https://cp3.irmp.ucl.ac.be/projects/madgraph/wiki/IntroMatching.

[38] J. Alwall et al., The automated computation of tree-level and next-to-leading order differential cross sections and their matching to parton shower simulations, JHEP 07 (2014) 079 [arXiv: 1405.0301] [INSPIRE].

[39] J. Pumplin, D.R. Stump, J. Huston, H.L. Lai, P.M. Nadolsky and W.K. Tung, New generation of parton distributions with uncertainties from global QCD analysis, JHEP 07 (2002) 012 [hep-ph/0201195] [INSPIRE].

[40] M. Cacciari, G.P. Salam and G. Soyez, FastJet User Manual, Eur. Phys. J. C 72 (2012) 1896 [arXiv: 1111.6097] [INSPIRE]. 